\title{
Cómo abordar el desarrollo de una competencia transversal compleja mediante el alineamiento constructivo.
}

\section{Isaias Sanmartin Santos ${ }^{\mathrm{a}}$, Ana Lloret Alcañiz ${ }^{\mathrm{b}}$, Ignacio Ventura González ${ }^{\mathrm{c}}$, Jesús Prieto Ruiz $^{\text {a }}$}

a. Departamento de Ciencias Médicas Básicas. Facultad de Veterinaria y Ciencias Experimentales. Universidad Católica de Valencia San Vicente Mártir.

b. Departamento de Fisiología. Facultad de Medicina. Universidad de Valencia.

c. Departamento de Ciencias Médicas Básicas. Facultad de Medicina. Universidad Católica de Valencia San Vicente Mártir.

\begin{abstract}
We present a model based on activities for the acquisition of essential competences of the scientific-technical qualifications, developed throughout the course of Cell Biology of the 1st year of the Degree in Biotechnology. For example, the search and analysis of bibliographic information and the design and interpretation of experiments are closely related and very important competences in the training of the biotechnologist, however they are not well contemplated in a specific way in the study plans. The proposed activities, designed under the premises of the constructive alignment of Biggs and Tang, develop these competences jointly and within the framework of a single subject.
\end{abstract}

Keywords: competences, training, teaching-learning activities, constructive alignment, design of experiments, interpretation of experiments

\begin{abstract}
Resumen
Presentamos un modelo basado en actividades para la adquisición de competencias esenciales de las titulaciones científico-técnicas, desarrollado a lo largo del curso de Biología Celular de $1^{\text {er }}$ curso del Grado en Biotecnología. Por ejemplo, la búsqueda y análisis de la información bibliográfica y el diseño e interpretación de experimentos son competencias intimamente relacionadas y muy importantes en la formación del biotecnólogo, sin embargo no se encuentran bien contempladas de forma específica en los planes de estudio. Las actividades propuestas, diseñadas bajo las premisas del alineamiento constructivo de Biggs y Tang, desarrollan estas competencias conjuntamente y en el marco de una única asignatura.
\end{abstract}

Palabras clave: competencias, formación, actividades de enseñanzaaprendizaje, alineamiento constructivo, diseño de experimentos, interpretación de experimentos 


\section{Introducción}

En los grados del ámbito de las ciencias básicas y biosanitarias, en particular en el Grado en biotecnología, resulta esencial desarrollar en nuestros alumnos competencias en la Búsqueda y análisis de la información bibliográfica y la Lectura e interpretación de artículos científicos.

También son de gran importancia las relacionadas con el Diseño y planificación de experimentos, y con la capacidad para Interpretar y discutir los datos experimentales obtenidos.

Por último, nuestros alumnos debieran adquirir durante el grado competencias relacionadas con la comunicación por escrito de los resultados de su trabajo práctico experimental, como los informes de resultados de un proyecto o las publicaciones científicas. Es decir, competencia en la Redacción de informes científicos .

Hemos observado que estas competencias se tratan por separado en los planes de estudio. Sin embargo a nivel profesional forman un "todo", ya que el biotecnólogo consulta la información bibliográfica disponible para planificar sus experimentos, analiza e interpreta los datos obtenidos, y publica sus resultados mediante informes de empresa o en revistas de investigación.

Se da también la situación paradójica de que, considerándose competencias transversales importantes y comunes a muchas asignaturas, precisamente por ello no se contemplan de manera específica en el plan de estudios. Su enseñanza se encuentra distribuida o fragmentada a lo largo de las prácticas de laboratorio y actividades de múltiples asignaturas, que tratan sólo con algunos aspectos parciales. A causa de ello hay un efecto de dilución o dispersión a lo largo de la titulación, no favorable para el buen aprendizaje de las competencias.

Además, consideramos que el fomento de estas competencias no debe focalizarse hacia el final del plan de estudios, sino que deben ser introducidas lo más tempranamente posible, ya que los alumnos se benefician de una adquisición temprana. Una introducción específica dedicada a desarrollarlas ya desde el primer curso, favorece una mejor asimilación de las múltiples asignaturas del Grado cuyos contenidos y actividades están relacionados con las competencias en el diseño e interpretación de experimentos

En el presente trabajo presentamos una propuesta de desarrollo integral de estas competencias a lo largo de una única asignatura, durante el primer curso del Grado en Biotecnología. La metodología elegida para desarrollar las competencias es el alineamiento constructivo. Según Biggs y Tang (Biggs, 2011) los objetivos de aprendizaje y la evaluación deben estar alineados con las actividades de enseñanzaaprendizaje. Hemos diseñado un modelo de adquisición de estas competencias 
siguiendo los principios del alineamiento constructivo, mediante una serie de actividades concatenadas en las que cada una desarrolla aspectos parciales para confluir en una gran actividad integradora final.

\section{Objetivos}

Nuestro objetivo es desarrollar las competencias en Búsqueda y análisis de la información bibliográfica, Diseño y planificación de experimentos, Interpretar y discutir datos experimentales y Redacción de informes científicos, pero de una forma integral e imbricada en los contenidos de una única asignatura. Para ello desarrollaremos una serie de actividades de enseñanza-aprendizaje relacionadas entre sí, y diseñadas bajo las premisas del alineamiento constructivo de Biggs y Tang.

\section{Desarrollo de la innovación}

En el presente trabajo, hemos logrado acoplar una serie de actividades relacionadas con competencias transversales de la titulación a los contenidos de una única asignatura. Para conseguirlo, ha habido que resolver una serie de problemas, relacionados con la complejidad de las competencias a desarrollar y las limitaciones de tiempo disponible. Para implementar este proyecto, ha sido necesario resolver los siguientes puntos clave:

I. Diseñar actividades de enseñanza-aprendizaje que pongan en juego los objetivos didácticos pretendidos. Siguiendo los principios del alineamiento constructivo, si nuestro objetivo es desarrollar competencias relacionadas con "saber hacer", debemos solicitar a nuestros alumnos una actividad evaluable que ponga en juego estas mismas competencias. Es decir, los alumnos deben hacer lo mismo que haría el biotecnólogo profesional, y el cómo de bien lo hagan es precisamente lo que se va a evaluar. La actividad elegida es "taller de aula virtual: informe final del experimento en el modelo C. elegans de anoxia/inanición". Esta actividad se realiza hacia el final del curso . En ella los alumnos elaboran un pequeño artículo científico muy sencillo, usando el juego de datos de un único experimento, representando una única figura y adicionando una pequeña bibliografía con sólo 2 o 3 artículos referenciados. Este experimento es el que planificaron y realizaron durante sus prácticas de laboratorio. Este artículo, pese a su sencillez, será elaborado siguiendo la estructura de redacción de las publicaciones científicas: título, resumen, introducción, resultados y discusión. Se requiere sólo un mínimo de 50-100 palabras para cada uno de estos apartados, lo cual es una extensión breve pero suficiente, y así se consigue que la carga de trabajo de la actividad sea asequible. 
II. Diseñar actividades complementarias que preparen para la actividad mayor final. La actividad descrita en el apartado anterior es compleja complejo, ya que implica o depende de muchas pequeñas habilidades diferentes. El alumno debe encontrar bibliografía adecuada en bases de datos de publicaciones científicas, para lo cual debe ser competente usando los motores de búsqueda, y también evaluando la información. Además, debe diseñar un experimento y ejecutarlo, representar graficamente los datos obtenidos, y obtener conclusiones acerca de ellos. Debe también organizar y describir sus hallazgos siguiendo las normas de redacción de artículos científicos, y otras varias habilidades que alumnos de primer curso sin experiencia no han adquirido. Por tanto, plantear directamente la actividad del apartado anterior, que integra aspectos de todas estas competencias, sería inapropiado y probablemente fracasaría en cuanto a sus objetivos educativos. En su lugar, hemos desarrollado una serie de actividades preparatorias o complementarias, que se realizan durante el curso, que desarrollan aspectos parciales y que converjen hacia la gran actividad integradora final del apartado anterior (Biggs y Tang, 2011).

III. Acomodar estas actividades en el plan de la asignatura. Otro problema adicional es el limitado tiempo disponible. La introducción de actividades para el desarrollo de estas competencias debe encajarse en el limitado número de sesiones de aula y laboratorio de la asignatura en la que se implemente el proyecto. Lo hemos solucionado de diversas maneras, por ejemplo proporcionando a los alumnos un juego de datos experimentales completos para la actividad final (evitando que tengan que obtener todos los datos ellos mismos en el laboratorio, lo cual consumiría un excesivo tiempo) o diseñando la actividad "taller hidromiel" de forma que participe toda la clase en un único gran experimento para la obtención de los datos experimentales (de esta manera el trabajo de obtención de los datos se reparte entre todos los alumnos, evitando el gran consumo de tiempo que conllevaría que cada alumno los obtuviese a nivel individual).

IV. Diseñar actividades que se relacionen directamente con los contenidos de la asignatura. No disponemos de una asignatura independiente dedicada a estos objetivos de aprendizaje, y todas las actividades anteriores deben encajarse en el contexto de los contenidos de la asignatura hospedadora. Lo hemos solucionado diseñando actividades a 
partir de los propios contenidos de la asignatura. Por ejempo, los casos de estudio utilizados (“experimentos clásicos en la biología molecular") son parte integral del programa de la asignatura. En lugar de estudiarlos teóricamente, se desarrollan como actividades de aula, y constituyen en sí mismos una introducción al Diseño e interpretación de experimentos. El diseño del experimento con C. elegans y la obtención de los datos necesarios para la gran actividad final, lo desarrollamos como una práctica de laboratorio en dos de las sesiones de prácticas de la asignatura. Y el taller de aula "proyecto hidromiel" se relaciona con los contenidos de la unidad didáctica dedicada a la mitocondria, en la que se explican las rutas fermentativas de obtención de energía en condiciones de anaerobiosis. Adicionalmente, este proceder representa una forma añadida de resolver el problema de la limitación de tiempo comentado en el punto anterior.

Para abordar el plan de actividades, sugerimos dividir la tarea en dos fases:

Primera fase. Definir los objetivos de aprendizaje que se pretenden conseguir. Deben situarse en un primer plano entre los objetivos de aprendizaje del curso y tener un peso específico en la asignatura, evitando así su consideración de competencia colateral o secundaria. Los situaremos en la guía docente. En nuestro caso, en la asignatura de biología celular del primer curso del Grado en Biotecnologia:

- una vez superado el curso, el estudiante debe ser capaz de buscar y analizar información científico-técnica en biología celular en bases de datos bibliográficas

- una vez superado el curso, el estudiante debe ser capaz de diseñar experimentos sencillos, analizar los datos e interpretar los resultados obtenidos

Segunda fase. Diseño, planificación y elaboración de las actividades de aula a través de las cuáles vamos a desarrollar la competencia, siguiendo los principios del alineamiento constructivo. Para el desarrollo de la competencia usaremos una evaluación formativa a través de una serie de actividades concatenadas, en las que cada actividad desarrolla un aspecto parcial, para confluir en una gran actividad integradora final.

a) Actividad de aula "demostración por el profesor de cómo se realiza una búsqueda bibliográfica"

Después de impartir la lección de aula "experimentos sobre vida artificial" se realiza la siguiente actividad demostrativa, que se anuncia a los alumnos como "búsqueda 
por el profesor de las publicaciones actuales de los experimentos sobre vida artificial del Instituto Venter y del grupo de investigación del profesor G.M. Church; lectura y revisión de los abstracts encontrados". El profesor muestra mediante el proyector del aula, cómo se obtienen publicaciones actualizadas sobre estos experimentos a través del motor de búsqueda PubMed, mostrándolo a los alumnos, a la vez que explica a los alumnos cómo hay que proceder en las búsquedas bibliográficas.

Qué aporta a la competencia Búsqueda y análisis de la información bibliográfica:

- los alumnos observan cómo se realiza una búsqueda en bases de datos de publicaciones científicas

- se introduce el concepto de keyword y cómo cruzarlas adecuadamente mediante los operadores lógicos AND, NOT y OR para realizar las búsquedas

- aprenden que el gran número de resultados que suelen aparecer en cualquier búsqueda debe acotarse con el uso de plabras clave adecuadas y operadores lógicos; asimismo deben filtrarse rápidamente a partir del título los artículos de interés, y en una segunda selección a partir de la lectura del abstract, en lugar de leer el artículo completo

\section{b) Seminario de aula "Estructura de un Artículo Científico"}

Se trata de un breve seminario de 15 minutos en el que se explican las partes constituyentes de un artículo científico (introducción, abstract, material y métodos, resultados y discusión) y qué tipo de información hay que situar en cada uno de estos apartados. Se proporciona a los alumnos un pequeño texto de apuntes como referencia. El profesor plantea preguntas a la clase y responde dudas.

Qué aporta a las competencias Búsqueda y análisis de la información bibliográfica y Redacción de informes cientificos:

- conocimiento acerca de la estructura de un artículo científico y qué tipo de información se encuentra en cada uno de los apartados

\section{c) Actividad de aula "interpretación de artículo científico "}

En esta actividad de aula, se proporciona a los alumnos un artículo científico impreso en papel y una lista de preguntas sobre este. El artículo elegido es "Condensation of an Additive-Free Cell Extract to Mimic the Conditions of Live Cells" (Fujiwara, 2013). Este artículo tiene relación directa con una de las actividades prácticas de laboratorio que realizamos más adelante. Las preguntas están clasificadas en función de cada apartado del artículo. Esta actividad es de larga duración, y requiere desarrollarla a lo largo de varias sesiones. Se acompaña de una presentación de diapositivas en las que se explica a los alumnos algunos de los métodos o 
instrumental científico utilizado en artículo, tan solo lo mínimo que se considere necesario para facilitar la comprensión. Son alumnos primerizos, que requieren de bastante ayuda por parte del profesor para que puedan acometer la actividad. A continuación, referimos a modo de ejemplo algunas de las preguntas de la actividad.

Introducción: ¿Cuál es el propósito de este trabajo? ¿Para qué resulta útil el método que se describe en él? ¿Por qué es un problema "the molecular crowding effect"?

Material y Métodos: ¿Qué significa "Green fluorescent protein (GFP) was overexpressed in E. coli"? ¿Por qué repiten varias veces cada experimento?

Resultados: ¿Qué aditivos son necesarios para conseguir que se produzca la expresión de proteínas en el sistema DDW? ¿Qué efecto causa sustituir el acetato potásico por glutamato? ¿Cómo lo explican?

Discusión: ¿Por qué piensan los autores que deben existir «factores adicionales aún desconocidos presentes en la célula viva», respecto a su sistema in vitro?

Qué aporta esta actividad a la competencia Búsqueda y análisis de la información bibliográfica:

- los alumnos examinan y leen por primera vez un artículo científico de cierto nivel de complejidad

- constituye un uso práctico del conocimiento adquirido en la actividad anterior sobre la estructura de un artículo científico

\section{d) Actividad de aula "experimentos clásicos en biología molecular"}

Esta actividad consiste en la realización de dos casos de estudio. Ha sido seleccionados porque están directamente relacionados con los contenidos teóricos de la asignatura, y porque además ilustran conceptos relacionados con el diseño e interpretación de experimentos. Ambos se encuentran disponibles en la web del National Center for Case Study Teaching in Science, University at Buffalo, en la ficha "Classic experiments in molecular biology" (Pals-rylaarsdam, 2014) .

- Caso de estudio I: Meselson and Stahl Experiment: The Nature of DNA Replication

- Caso de estudio 2: The Transforming Principle: Identifying the Molecule of Inheritance

Los dos casos tratan acerca de la naturaleza del descubrimiento científico, el diseño de experimentos, el uso de controles exprimentales, y la lógica o interpretación de los resultados de experimentos. Se relacionan directamente con el cuerpo de 
contenidos de la asignatura, lo cual es ventajoso en dos sentidos: son seguidos con mayor interés por los alumnos porque no los consideran un anexo sino parte integral de la asignatura, y el gasto de tiempo de aula implicado es menor porque se trata de la propia materia del curso (y no de actividades extra no relacionadas directamente con los contenidos del programa).

Los alumnos resuelven los casos en el aula, organizados en grupos de trabajo de cuatro alumnos, y apoyados por el profesor. Cada alumno entrega sus propias respuestas, que serán evaluadas. La retroalimentación o "assesment" se realiza durante y al final de la propia sesión, explicando a todos los alumnos del aula las dudas eventualamente planteadas por algún alumnos y aclarando cuáles son las respuestas correctas mediante un diálogo compartido entre la clase completa y el profesor, a la manera de un seminario. No es un objetivo que el alumno encuentre las respuestas correctas del caso de estudio de forma individual, sino que estas se consensuan entre el grupo de trabajo y, antes de finalizar la actividad, con el propio profesor (en un diálogo abierto con toda la clase). Se pretende tan solo que el alumno entienda bien el caso de estudio y sea capaz de redactar correctamente sus respuestas.

Qué aporta a las competencias Diseño y planificación de experimentos, Interpretar $y$ discutir datos experimentales:

- introduce al estudiante al diseño de experimentos y a su interpretación

- introduce conceptos como "controles experimentales" y a cómo se resuelven preguntas científicas críticas mediante un experimento

\section{e) Taller de aula "Proyecto hidromiel"}

El objetivo de este taller es proporcionar una introducción al diseño y realización de experimentos. La primera parte comienza en el aula. Se entrega un pequeño biorreactor a cada alumno, y varias cepas de levadura industrial de comportamiento fermentativo distinto (una fermenta más rápidamente, otra consigue niveles más elevados de alcohol, etc.). Deben encontrar condiciones adecuadas para una fermentación de hidromiel que rinda un producto óptimo, de características predefinidas por el profesor. Se les pide que encuentren condiciones como cantidad de levadura inicial, la proporción agua:miel de la mezcla, etc. para obtener un producto de $10^{\circ}$ de alcohol en un tiempo de fermentación de 7 días y con cierta cantidad de azúcares residuales para lograr un sabor dulce. Estas condiciones deben hallarlas realizando un experimento masivo con los biorreactores proporcionados.

Teniendo en cuenta que son alrededor de 60 alumnos, pueden realizarse hasta 60 experimentos individuales. Los alumnos se organizan en grupos de 4-6 para diseñar un gran experimento conjunto en el que cada alumno ensayará personalmente unas condiciones de fermentación. El grupo debe entregar una hoja con el diseño 
experimental, que será evaluada y sumará para la nota final de actividades de la asignatura.

En una segunda parte que se realiza en el laboratorio, cada alumno prepara sus biorreactores según las condiciones elegidas. Recogen a partir de ellos los datos experimentales durante una semana, y posteriormente los representan gráficamente para decidir cuáles son las mejores condiciones para obtener un hidromiel de las características requeridas. Deben presentar un informe de resultados grupal con estas gráficas y conclusiones, que también será evaluado. La actividad finaliza con un cuestionario de tipo test que relaciona el taller hidromiel con los contenidos del tema sobre la mitocondria del libro de texto de base de la asignatura. Este test se contesta en el aula virtual Moodle de la asignatura.

Qué aporta a las competencias Diseño y planificación de experimentos e Interpretar $y$ discutir datos experimentales:

- los alumnos aprenden a diseñar un experimento

- ejecutan de manera real el experimento

- grafican e interpretan los resultados obtenidos

f) Práctica de laboratorio "Construcción de una célula artificial para síntesis de proteínas de interés biotecnológico”

Se trata de reproducir un protocolo experimental de cierta complejidad, adaptado a la disponibilidad de tiempo y materiales del laboratorio de prácticas, y a las posibilidades de los alumnos del primer curso (todavía con escasa experiencia de trabajo en el laboratorio). A lo largo de varias sesiones prácticas los alumnos trabajan con varios protolos experimentales simplificados para la obtención de un producto biotecnológico final (una suspensión de liposomas que encapsulan la maquinaria de transcripción-traducción de bacterias para síntesis de proteínas in vitro).

La práctica se basa en el artículo "The Encapsulation of Cell-free Transcription and Translation Machinery in Vesicles for the Construction of Cellular Mimics", de la revista The Journal of Visualized Experiments que se caracteriza porque cada artículo lleva asociado un reportaje grabado en video. El primer paso es la visualización del vídeo en el que se basa la práctica. Se realiza en el aula, comentando el artículo asociado, aclarando los aspectos difíciles y resolviendo las eventuales dudas.

En segundo lugar, cada subgrupo de alumnos reproduce este trabajo durante varias sesiones prácticas de laboratorio. En el artículo original, utilizan un reactivos comerciales basado en ribosomas purificados (PURExpress ${ }^{\circledR}$, de New England Biolabs). Este producto es demasiado caro, por lo que en su lugar usamos el extracto DDW de E. coli publicado en el artículo utilizado para la actividad "interpretación 
de artículo científico", que puede sustituirlo, ya que tiene una acción equivalente. De esta manera, enlazamos dos actividades (lo cual a nuestro juicio tiene un efecto pedagógico superior) y conseguimos un ahorro importante tanto en el tiempo de aula dedicado a la actividad como en el coste de tiempo para el alumno (ya que los protocolos ya se explicaron en la actividad mencionada).

Adicionalmente, esta actividad genera aprendizajes muy interesantes, pues muestra cómo ir un paso más allá de un protocolo propuesto en un artículo para proponer un desarrollo nuevo, lo cual está en el corazón de las competencias necesarias a adquirir por un biotecnólogo en formación.

Qué aporta a las competencias Diseño y planificación de experimentos e Interpretar y discutir datos experimentales:

- los alumnos realizan una versión simplificada de un protocolo completo real para el desarrollo de un producto biotecnológico

- enlazan esta actividad con la de "interpretación de un artículo científico" para desarrollar un producto alternativo nuevo, lo que genera aprendizajes interesantes y muy motivadores sobre el uso de la bibliografía científica en biotecnología

g) Práctica de laboratorio "Modelo de isquemia/reperfusión por anoxia/inanición en C. elegans y acondicionamiento preanestésico"

A lo largo de dos sesiones prácticas de laboratorio, los alumnos diseñan un experimento para testar una sustancia con posible efecto protector celular, a partir del modelo presentado en el viedo-artículo "An anoxia-starvation model for ischemia/reperfusion in C. elegans" (Queliconi, 2014). En primer lugar, se visualiza en el aula el video que acompaña el artículo. A continuación, se asigna a cada subgrupo de alumnos una sustancia a testar (fármacos, vitaminas, etc.) y diseñan el experimento ayudados por el profesor. Durante dos sesiones prácticas de laboratorio realizarán el experimento y obtendrán los resultados asociados. El tiempo disponible es insuficiente para un experimento real, así que sólo obtienen unos pocos datos.

Qué aporta a las competencias Diseño y planificación de experimentos e Interpretar y discutir datos experimentales:

- los alumnos diseñan y realizan su propio experimento

- obtienen datos experimentales reales a partir de este 
h) Taller de plataforma "Informe final del experimento en el modelo $C$. elegans de anoxia/inanición”

Esta es la actividad final y la más importante, pues representa la culminación del proceso y que mostrará la adquisición de las competencias.

Los alumnos deben redactar un artículo de investigación sobre lo obtenido en el experimento con $C$. elegans, muy breve pero con la estructura completa: título, resumen, introducción, resultados, discusión, bibliografía. Se utiliza una plantilla en la que se les indica las limitaciones de cada apartado (por ejemplo, 50-100 palabras para la introducción, 1 o 2 figuras como máximo para los resultados, y un máximo de 3 citas bibliográficas). Deben subir su trabajo como una tarea Moodle al aula virtual de la asignatura para su evaluación.

Se complementa la actividad con un tutorial acerca de la graficación e interpretación de datos, y tutoriales online sobre el uso de Excel para graficar datos.

La evaluación de esta competencia sigue puramente los principios del alineamiento constructivo: para enseñar a diseñar e interpretar experimentos y escribir reportes científico-técnicos sobre los resultados, pedimos a nuestros alumnos que diseñen e interpreten un experimento y redacten un pequeño artículo científico final. Serán evaluados en cuanto a cuan bien haya sido realizado este reporte final.

Qué aporta a las competencias Búsqueda y análisis de la información bibliográfica, Diseño y planificación de experimentos e Interpretar y discutir datos experimentales y Redacción de informes científicos:

- los alumnos escriben un pequeño informe científico sobre el experimento que ellos mismos planificaron y realizaron en sus prácticas de laboratorio, realizando de forma completa una tarea compleja que requiere de la adquisición previa de competencias parciales adquiridas a través de las actividades anteriores (búsqueda de información bibliográfica, diseño de experimentos, interpretación de resultados experimentales, etc.)

\section{Resultados}

Los problemas con los que nos hemos encontrado al tratar de incorporar estas competencias transversales en una única asignatura son fundamentalmente la limitación de tiempo y la propia complejidad para los alumnos de las competencias.

Para solucionar el problema del tiempo, hemos diseñado actividades que se alinean directamente con los contenidos de la asignatura. Un ejemplo serían los casos de estudio sobre los experimentos clásicos en biología molecular. Una ventaja adicional de esta solución es que los alumnos contemplan estas actividades como parte integral 
del programa de la asignatura Biología Celular de la que van a examinarse, y por tanto las siguen con mayor interés y dedicación que si las consideran algo colateral o un anexo a la asignatura. Por otro lado, las actividades están relacionadas unas con otras, de manera que se abrevia mucho el tiempo de explicación y preparación de la nueva actividad si la basamos en el material de una actividad anterior. Por ejemplo, el protocolo para preparar el extracto DDW de E. coli de la práctica de generación de "células artificiales", procede del artículo que se utiliza en la actividad de aula "interpretación de artículo científico ".

Otra solución que permite abordar las actividades en un tiempo razonable es el diseño o adaptación de los experimentos prácticos. Por ejemplo, a diferencia de la preparación real del hidromiel, que requiere dos fermentaciones sucesivas del producto, en el proyecto hidromiel se utiliza tan solo una única fermentación pues es más que suficiente para nuestro propósito. Aunque no se obtendrá un hidromiel con buenas propiedades organolépticas, para nuestro proyecto este aspecto no es relevante. Por otro lado, en las prácticas con C. elegans, la toma de datos experimentales no es completa. Aunque el experimento se realiza fielmente, la obtención de resultados (observación de los nematodos) requiere de muchas horas, lo cual es inasumible en el marco de unas prácticas de laboratorio normales (que representan aproximadamente tan solo un 15\% del tiempo presencial de la asignatura) . Por ello sólo se piede a los alumnos que observen y registren algunos datos a nivel testimonial. Al finalizar la sesión de laboratorio, el análisis de resultados no es sobre sus propios datos sino sobre un juego de datos experimentales completo proporcionado por el profesor. En la práctica de laboratorio de construcción de células artificiales, se consigue abreviar su duración de la siguiente manera: aunque los alumnos realizan por ellos mismos casi todos los pasos experimentales (es un protocolo largo que se prolonga durante varias sesiones) los pasos clave son realizados a nivel demostrativo por el profesor. Y el protocolo de extracción de la maquinaria de transcripción-traducción de bacterias utilizado procede del artículo científico de la actividad de aula "interpretación de artículo científico", con lo que los alumnos ya lo conocen y no se requiere de tiempo de explicación adicional.

Para solucionar el problema de la complejidad de las competencias tratadas, proponemos realizar una serie de actividades concatenadas que van educando a lo largo del curso en los aspectos parciales, y que confluyen en una gran actividad integradora final.

La evaluación de estas actividades sigue en la medida de lo posible las premisas fundamentales del alineamiento constructivo de Biggs y Tang: la evaluación debe estar alineada con las actividades de enseñanza-aprendizaje, y ambas dirigidas a su 
vez a conseguir los resultados u objetivos pretendidos de aprendizaje. Así por ejemplo, la evaluación de la última actividad implica la redacción de un pequeño artículo científico y es por tanto el punto final en el que confluyen la actividad acerca de cómo realizar búsquedas bibliográficas, el taller de aula proyecto hidromiel (que enseñaba a diseñar e interpretar experimentos) y la práctica de laboratorio con $C$. elegans. Al elaborar este artículo científico final, los alumnos ponen en juego y evidencian los resultados de aprendizaje que adquirieron en las actividades de enseñanza-aprendizaje que realizaron durante el curso.

\section{Conclusiones}

El desarrollo de las competencias Búsqueda y análisis de la información bibliográfica, Diseño y planificación de experimentos, Interpretar y discutir datos experimentales y Redacción de informes científicos, puede implementarse de manera conjunta e integral a lo largo de una única asignatura. Tal desarrollo puede basarse en actividades concatenadas en las que se adquieren progresivamente las competencias. Hemos diseñado nuestras actividades bajo las premisas del alineamiento constructivo de Biggs y Tang y relacionándolas en la medida de lo posible con los contenidos del programa la asignatura. El problema del excesivo consumo de tiempo por estas actividades puede gestionarse adecuadamente si se alinean con los contenidos teóricos del curso, ensamblando las actividades de manera que cada nueva actividad se base o apoye en una de las anteriormente realizadas, y modificando las actividades prácticas de laboratorio de forma que consuman un menor gasto de tiempo para realizarlas. La intervención, aunque presumiblemente mejora las competencias tratadas, no puede valorarse actualmente con datos objetivos. Por ejemplo, puede intentar comprobarse si ha habido una mejora en las calificaciones obtenidas en las actividades relacionadas con las competencias, pero solo en los años venideros en los que los que los alumnos que pasaron por nuestra asignatura se enfrenten a actividades de cursos superiores que pongan en juego estas competencias . Por otro lado, tampoco podemos obtener datos válidos con los que juzgar la bondad de la intervención a partir de las opiniones o encuestas de satisfacción de los alumnos, ya que se trata de alumnos nuevos recién llegados a la universidad, por lo que no tienen un bagaje o experiencia previa acumulada frente al que referenciar o contrastar su experiencia en nuestra asignatura. No obstante, el alineamiento constructivo ha demostrado ampliamente su eficacia como metodología docente en la universidad, y creemos que su implementación indudablemente contribuye a mejorar la educación en nuestras aulas.

\section{Referencias}

BIGGS J, TANG C. (2011) Teaching for Quality Learning at University. Society for Research Into Higher Education: London, Open University Press; 4 edition. 
FUJIWARA K, NOMURA SM. (2013) "Condensation of an additive-free cell extract to mimic the conditions of live cells". PLoS One. 8(1):e54155. doi: 10.1371/journal.pone.0054155. Epub 2013 Jan 10.

LI J, GU L, AACH J, CHURCH GM (2014) “Improved Cell-Free RNA and Protein Synthesis System” PLoS ONE 9(9): e106232.

PALS-RYLAARSDAM, R., Classic Experiments in Molecular Biology. National Center for Case Study Teaching in Science, University at Buffalo, New York. http://sciencecases.lib.buffalo.edu/cs/collection/detail.asp?case_id=641\&id=641> [Consulta: 07 de septiembre de 2014].

QUELICONI, BB., KOWALTOWSKI, AJ y NEHRKE, K., (2014). “An anoxiastarvation model for ischemia/reperfusion in C. elegans”. En J Vis Exp. 11;(85).

SPENCER AC1, TORRE P, MANSY SS. "The encapsulation of cell-free transcription and translation machinery in vesicles for the construction of cellular mimics.” J Vis Exp. 2013 Oct 21;(80):e51304. 